\title{
Commentary: Diastolic dysfunction following biventricular conversion: When physiology beats anatomy
}

\author{
Bahaaldin Alsoufi, MD
}

\author{
From the Department of Cardiovascular and Thoracic Surgery, University of Louisville School of Medicine, \\ Louisville, Ky. \\ Disclosures: Author has nothing to disclose with regard to commercial support. \\ Received for publication Sept 17, 2019; revisions received Sept 17, 2019; accepted for publication Sept 17, 2019; \\ available ahead of print Oct 24, 2019. \\ Address for reprints: Bahaaldin Alsoufi, MD, Department of Cardiovascular and Thoracic Surgery, University of \\ Louisville School of Medicine, Norton Children's Hospital, 201 Abraham Flexner Way, Suite 1200, Louisville, \\ KY 40202 (E-mail: balsoufi@ hotmail.com). \\ J Thorac Cardiovasc Surg 2020;159:647-8 \\ $0022-5223 / \$ 36.00$ \\ Copyright (c) 2019 by The American Association for Thoracic Surgery \\ https://doi.org/10.1016/j.jtcvs.2019.09.104
}

A child was born with borderline left ventricle, mitral, and aortic valves. He underwent first-stage palliation followed by Glenn. Before Fontan, he was considered for biventricular conversion. Subsequently, he underwent 3 operations toward achieving that goal. Those entailed atrial septal defect closure, takedown of Damus-Kaye-Stansel, septal myectomy, resection of endocardial fibroelastosis (EFE), aortic commissurotomy, ascending aorta patch, mitral valve repair followed by replacement, pulmonary artery patch, and takedown of Glenn with superior vena cava patch. Although the size of his left-sided structures at that point seemed adequate, and systolic function was normal, his recovery was prolonged and complicated. Invasive and noninvasive studies demonstrated significant diastolic dysfunction and pulmonary hypertension that improved over few months, allowing hospital discharge. Nonetheless, he was readmitted 2 months later with heart failure and diastolic dysfunction. Despite the challenges of mechanical support in patients with diastolic dysfunction, he had left and right ventricular assist device implantation due to evolving end-organ failure. While clinically improved, his transplant waiting-list time was long (due to sensitization), and he developed embolic complications and subsequently died.

This story unfortunately sounds familiar to many surgeons and is testament of our continuous struggle assessing and managing diastolic dysfunction in children with complex congenital heart disease. The question of whether a very complex biventricular repair in children with borderline ventricle is better than a simple single-ventricle palliation is still unresolved. If we had selected to continue with the single-ventricle strategy, this child would have done very well for many years following Fontan. Yet, we felt compelled to recommend biventricular conversion, which is associated with complicated operations and their attendant morbidity and mortality risks, all with the expectation to avoid long-term complications of Fontan physiology. Although discouraging incidents like this should not deter

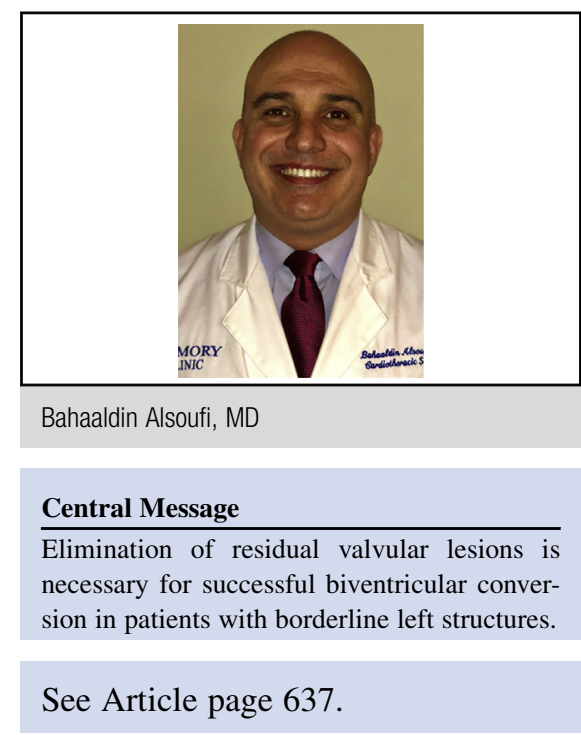

us from pursuing better physiologic repairs, they certainly incite us to explore ideal candidates and best operations for those patients.

The Boston group has championed left ventricle recruitment and biventricular conversion strategies and has contributed a great deal to our knowledge related to this matter. $^{1-3}$ They introduced staged left ventricular recruitment to progressively increase left ventricle loading and they demonstrated subsequent growth allowing successful biventricular conversion. ${ }^{1,2}$ They also demonstrated that although growth in size was necessary, it was not always enough for predicting successful biventricular conversion and that diastolic dysfunction was a major problem in some of those patients. ${ }^{3}$ They demonstrated that elevated end-diastolic pressure (left ventricular enddiastolic pressure [LVEDP]) $>13 \mathrm{~mm} \mathrm{Hg}$ and ensuing elevated postoperative right ventricle pressure $>3 / 4$ systemic were associated with biventricular conversion failure and death. ${ }^{3}$ They also determined that patients with hypoplastic left heart syndrome (HLHS) did worse than those with unbalanced atrioventricular septal defects and postulated that intrinsic myocardial fibrosis and EFE in patients with HLHS were related to elevated LVEDP and suggested that redo EFE resection and repair of residual mitral/aortic stenosis or regurgitation might be beneficial in those patients. $^{3}$

In the current issue of the Journal, the Boston group offers another contribution to this field, and they focus on pathophysiology of EFE and its association with residual 
valvular flow disturbances (stenosis or regurgitation). ${ }^{4}$ They analyzed EFE tissues from 24 patients with HLHS who underwent left ventricle recruitment and compared them with a control group of 6 patients without HLHS with flow disturbances across aortic and/or mitral valve who had the macroscopic appearance of EFE-like tissue identified during their cardiac surgery.

The authors concluded that valvular flow disturbances expose endocardial endothelial cells to altered shear forces and consequently induce endothelial-to-mesenchymal transition leading to EFE recurrence that displayed a more infiltrative growth pattern into the underlying myocardium (difficult to separate from underlying myocardium) and that this process is related closely to valvular flow disturbances and not necessarily to underlying cardiac defect (HLHS). ${ }^{4}$ The simple clinical inference from that study is that surgeons should aim to eliminate any significant residual valvular lesion during left ventricle recruitment procedures, along with EFE resection, with the objective of optimizing diastolic ventricular function and achieving good biventricular conversion outcomes.

That notion would propose several changes to left ventricle recruitment strategies that include the following: (1) the number of procedures necessary before completing biventricular conversion (eg, patients with persistent elevated LVEDP and residual valvular lesions might require intermediary redo aortic/mitral valve repair/replacement and repeat EFE resection); (2) type of the procedures performed to achieve best outcomes (eg, Ross procedure or mitral valve replacement might be better than aortic/mitral valve repair with hemodynamically-significant residual lesion); (3) timing of procedures performed for left ventricle recruitment (eg, most patients undergo aortic/ mitral valve repair and EFE resection during Glenn, better repair might possibly be achieved at an older age); and (4) candidacy for left ventricle recruitment (eg, patients expected to continue to have diastolic dysfunction despite mitral valve replacement are likely better served staying with single-ventricle palliation). In addition, further research is necessary to improve our understanding of EFE and myocardial fibrosis pathophysiology, to expand our ability to diagnose myocardial fibrosis and assess diastolic dysfunction, and to explore potential local treatments that might alter the genesis of EFE and myocardial fibrosis.

In summary, we should continue to push boundaries to achieve biventricular repair in patients with borderline left heart structures. We know for sure that size alone is not enough to predict successful biventricular repair, and that diastolic dysfunction is the main problem affecting repair outcomes. In those patients, physiology beats anatomy, and disappointing outcomes can be achieved in patients with bad physiology despite good systolic function and adequate-size structures. Better tools to assess and treat diastolic dysfunction and altered management strategies to eliminate any significant residual flow disturbances might further refine our efforts to safely achieve durable biventricular repair that is associated with less reoperation requirements and better functional status of the patients.

\section{References}

1. Emani SM, McElhinney DB, Tworetzky W, Myers PO, Schroeder B, Zurakowski D, et al. Staged left ventricular recruitment after single-ventricle palliation in patients with borderline left heart hypoplasia. J Am Coll Cardiol. 2012;60: 1966-74.

2. Banka P, Schaetzle B, Komarlu R, Emani S, Geva T, Powell AJ. Cardiovascular magnetic resonance parameters associated with early transplant-free survival in children with small left hearts following conversion from a univentricular to biventricular circulation. J Cardiovasc Magn Reson. 2014;16:73.

3. Herrin MA, Zurakowski D, Baird CW, Banka P, Esch JJ, del Nido PJ, et al. Hemodynamic parameters predict adverse outcomes following biventricular conversion with single-ventricle palliation takedown. J Thorac Cardiovasc Surg. 2017;154: 572-82.

4. Weixler V, Marx GR, Hammer PE, Emani SM, del Nido PJ, Friehs I. Flow disturbances and the development of endocardial fibroelastosis. J Thorac Cardiovasc Surg. 2020;159:637-46. 